\title{
Absolute identification of two-dimensional tones
}

\author{
ANTE FULGOSI, DJURDJICA BAČUN, and BOŽO ŽAJA \\ Odsjek za psihologiju, Filozofskifakultet, 41000 Zagreb, Yugoslavia
}

\begin{abstract}
By combining seven frequencies (between $250 \mathrm{~Hz}$ and 3,000 Hz) with four adequately selected intensity levels, 28 stimuli differing simultaneously in pitch and loudness (four loudness levels) were obtained. Stimuli were presented to five subjects 75 times each in an absolute judgment paradigm. In the last third of the experiment, median transmission value was 3.85 bits (between 14 and 15 categories). The amount of transmitted information in each dimension was smaller than the corresponding values in unidimensional transmission experiments. The difference in the identification ability of pitch and loudness in one- and two-dimensional situations is attributed to interference which is present in one and absent in the other situation and which may indicate differences in the encoding processes of one- and two-dimensional tones.
\end{abstract}

In a recent investigation of the amount of transmitted information in absolute identification of pure tones differing only in frequency, Fulgosi and Żaja (1975) have obtained 3.1 bits as the median value of transmission. This value is equal to the amount of transmitted information which Pollack (1953) obtained for twodimensional tones.

It is well known that the amount of information transmitted in absolute identification of stimuli depends to a large extent on the dimensionality of stimuli which are presented for identification. For this reason, it seems reasonable to expect that the ability of subjects to identify two-dimensional tones (tones differing in pitch and loudness for example) should be better than their ability to identify one-dimensional tones. Since, in the earlier investigation of Pollack (1953) with two-dimensional tones and the recent investigation of Fulgosi and Żaja (1975) with one-dimensional tones, the amount of transmitted information was the same, it seems necessary to investigate once more the ability of identification of tones differing in pitch and loudness.

Pollack (1953) presented for identification 25 tones obtained by combination of five intensities and five frequencies. Frequencies were selected between $125 \mathrm{~Hz}$ and $7,000 \mathrm{~Hz}$ in equal logarithmic steps. Five intensities were in equal intervals between 20 and $90 \mathrm{~dB}$. Results were analyzed from the whole range of experiments. Pollack does not mention how many times each tone was presented for identification, but, from the fact that all responses were analyzed together, it seems that the number of times each tone was presented was not very large.

\section{METHOD}

\section{Subjects}

Five undergraduate students in psychology served as subjects in the experiment. All had normal hearing, and all were naive about the purpose of the experiment.

\footnotetext{
Apparatus

Tones were generated by a beat/frequency oscillator (Bruel \& Kjaer, Type 1022) connected with a verbo-tonal audiometer.
}

Table 1

Combinations of Frequencies and Intensity Levels

\begin{tabular}{ccccc}
\hline & \multicolumn{4}{c}{ Intensity Levels in dB } \\
\cline { 2 - 5 } $\begin{array}{c}\text { Frequencies } \\
\text { in Hz }\end{array}$ & I & II & III & IV \\
\hline 250 & 60 & 83 & 95 & 105 \\
500 & 45 & 82 & 95 & 105 \\
750 & 45 & 82 & 95 & 105 \\
1000 & 37 & 82 & 95 & 105 \\
1500 & 39 & 82 & 95 & 105 \\
2000 & 39 & 82 & 95 & 105 \\
3000 & 50 & 83 & 98 & 105 \\
\hline
\end{tabular}

This set was connected with five Koss earphones ( $6 \mathrm{ohms}$ ).

A timer which automatically controlled duration of the stimuli and the interstimulus interval was included in the circuit.

\section{Stimuli}

Twenty-eight tones, differing in frequency and intensity, served as stimuli. Stimuli were obtained by combining seven frequencies and four intensity levels for each frequency according to Table 1 .

Intensity levels and frequencies were combined in such a way as to keep the loudness (subjective experience of intensity) of different frequencies as equal as possible. For this purpose, the table of tones appearing in Stevens (Licklider, 1951, p. 1002) was used.

Duration of each stimulus was $5 \mathrm{sec}$, and the interstimulus interval was also $5 \mathrm{sec}$.

\section{Procedure}

Experiment was composed of two parts. In the first part, the seven frequencies were presented on two intensity levels (two middle columns of intensities in Table 1). In the second part, all four intensity levels were used (28 stimuli).

The task of subjects in the first part of the experiment was to identify each stimulus with two numbers. The first number was for loudness and could be 2 or 3 . The second number was for pitch and could be between 1 and 7 , since seven pitches appeared.

In the second part, subjects were instructed to use numbers from 1 to 4 for the identification of loudness.

There were six experimental sessions in the first part of the experiment (two in each day). Each session lasted about $1 \mathrm{~h}$. During each session, each stimulus was presented 10 times in random order, which means that each subject gave 840 identification responses (140 identification responses in each session). 
Table 2

Amount of Information Transmitted in the First Part of the Experiment

\begin{tabular}{ccc}
\hline & \multicolumn{2}{c}{ Halves } \\
\cline { 2 - 3 } Subject & I & II \\
\hline 1 & 2.79 & 3.30 \\
2 & 2.38 & 2.60 \\
3 & 2.33 & 2.77 \\
4 & 2.32 & 2.44 \\
5 & 2.15 & 2.86 \\
\hline
\end{tabular}

Note-Italicized values represent medians.

Table 3

Amount of Information Transmitted in the Second Part of the Experiment

\begin{tabular}{cccc}
\hline & \multicolumn{3}{c}{ Thirds } \\
\cline { 2 - 4 } Subject & I & II & III \\
\hline 1 & 4.23 & 4.31 & 4.37 \\
2 & 3.46 & 3.63 & 3.93 \\
3 & 3.41 & 3.56 & 3.60 \\
4 & 3.03 & 3.29 & 3.37 \\
5 & 3.47 & 3.74 & 3.85 \\
\hline
\end{tabular}

Note-Italicized values represent medians.

Table 4

Information Transmitted in the Last Third of the Experiment in Each Dimension

\begin{tabular}{cccc}
\hline Subject & Loudness & Pitch & Sum \\
\hline 1 & 1.84 & 2.33 & 4.17 \\
2 & 1.76 & 1.94 & 3.70 \\
3 & 1.69 & 1.65 & 3.34 \\
4 & 1.49 & 1.53 & 3.02 \\
5 & 1.60 & 1.91 & 3.51 \\
\hline
\end{tabular}

Note-Italicized values represent medians.

There were 15 experimental sessions in the second part of the experiment, two each day. Each session lasted about $1 \mathrm{~h}$. Each stimulus appeared five times during a session, in random order, which means that in the second part of the experiment each subject gave 2,100 identification responses (140 identification responses in each session).

Before the beginning of the experiment and before the beginning of each session, the experimenter presented all frequencies in the first, and after that in the second, intensity level (in the second part of the experiment, in four intensity levels) and gave the right identification numbers for loudness and pitch for that stimulus.

For the purpose of training in the procedure, 14 stimuli used in the first part of the experiment were presented to subjects in random order 10 times. Subjects were asked to identify each stimulus with the proper numbers and to write their answers in protocols. After each presentation and after subjects wrote their answers, the experimenter gave the right identification numbers for that stimulus. Subjects were asked to mark each answer which was right (separately for loudness and pitch). At the end of each experimental session, they were asked to count these marks (separately for loudness and pitch).

\section{RESULTS}

Amount of transmitted information was calculated for each subject.

In the first part of the experiment, transmission values were calculated for the first and the second halves of this part of the experiment (for the first 420 and the second 420 identification responses) and appear in Table 2.

As can be seen, all subjects transmitted more information in the second than in the first half. Median value in the first half corresponds to five categories and median value in the second half to seven categories.

Amounts of transmitted information in the second part of the experiment were calculated for each third of the experiment (Fulgosi and Bartolovic, 1971) or for each 700 successive presentations. Results appear in Table 3.

Table 3 shows that all subjects advanced in the identification performance during this part of the experiment. Median value in the first third of this part corresponds to 11 categories, and the median value in the last third to 14.5 categories.

From the responses in the last third of the second part of the experiment, separate amounts of transmitted information for pitch and loudness were calculated. These values appear in the Table 4.

As can be seen from Table 4, the median transmission for intensity is 1.69 bits, and for frequency 1.91 bits. The maximum possible transmission values for these two dimensions are 2 and 2.8 bits, respectively.

Information transmission values for each subject in the last third of the experiment when the two dimensions are taken together (Table 3) are larger than the comparative sums of transmitted information in each of the two dimensions (Table 4).

\section{DISCUSSION}

As can be seen from Table 3, in the last third of the experiment, subjects were able to identify more than 14 tones, differing simultaneously in pitch and loudness, much more (67\% more) than Pollack (1953) obtained for two-dimensional tones and more than Fulgosi and Žaja (1975) obtained for one-dimensional tones.

The difference can be attributed to differences between the earlier and the present two-dimensional experiments.

Subjects in the present experiment had probably a larger amount of training than subjects in the earlier one. The ways the transmitted information was calculated in the two experiments is also different. In the first experiment, the transmission indexes were calculated from the whole range of the experiment. In the present experiment, they were calculated separatedly for different phases. In such calculation, transmission values are not contimated by identification errors which subject made in earlier phases and which, in an artificial way, enlarge the amount of equivocation and reduce the transmission (Fulgosi \& Bartolovic, 1971).

It should be pointed out that the range of frequencies in the present experiment was only half the range which Pollack used (Pollack, 1953). But, frequencies in the present experiment were combined with intensities in such a way as to keep the subjective intensity (loudness) of tones differing in frequency as much equal as possible. Pollack (1953) kept the intensity level equal for all frequencies which were used. Since loudness of tones depends not only on intensity but on the combination of intensity and frequency, subjects in the earlier experiment probably experienced more intensities than they were told they 
would have. Such a situation could easily produce more identification errors.

In the present experiment, as well as in the earlier experiments in which more than one dimension was used, the sum of the amounts of transmitted information in each dimension appeared smaller than the amount of transmitted information calculated from two-dimensional stimuli. Median values in Table 4 , when summed, give only 3.60 bits $(1.69+1.91)$ vs. the median of 3.85 bits in Table 3. The same relation holds for each subject. This shows that there exists an interaction between the two dimensions.

Since the informational value of the set of 28 stimuli used in the present experiment was 4.8 bits, it is clear that the results were not limited by the amount of uncertainty of the stimulus set. On the other hand, it is difficult to believe that much more improvement in the identification could be expected from the continuation of training in the present experiment. This does not mean that a different combination of pitches and loudness or a different number of categories in each dimension could not bring the identification performance to an even higher value. But to achieve this, in our opinion, such a combination should, if possible, produce a smaller amount of mutual interference between the judgments of pitch and loudness than it did in this experiment.

That a strong interference of the two judgments existed in the present experiment can be seen from the fact that our subjects have not reached the maximal values of transmission in each dimension which were possible ( 2 bits for loudness and 2.8 bits for pitch), in spite of the fact that these values are lower than the maximal transmission values found in unidimensional transmission experiments: 2.1 bits for loudness (Garner, 1953) and 3.1 bits for pitch (Fulgosi and Žaja, 1975). Detrimental effect of interference is especially evident in the dimension of pitch.

Explanation of the smaller transmission values per dimension in terms of interference seems to us more plausible than the explanation in terms of larger memory load in the situation of two-dimensional stimuli, since the duration of stimuli in the present experiment was long enough to allow the subjects to make separate judgments of loudness and pitch while the tone was still lasting.

The interference of the two kinds of judgments, as well as the difference between the sums in Table 4 and the transmission values in Table 3 , seems to point to differences in the encoding processes which may exist with one- and two- dimensional tones. It seems that the two-dimensional tones are encoded as perceptual units.

In such units, the two dimensions-pitch and loudness-are fused; and, therefore, it is more difficult to make judgments about each component than is the case with one-dimensional tones. It seems that separate judgments about one component requires a previous decomposition of each perceptual unit and that the training influence the easiness and precision of subjects in making such decomposition.

\section{REFERENCES}

Fulgosi, A..\& Bartolovíc, B. Practice and some methodological problems in unidimensional information transmission. Perception \& Psychophysics. 1971, 10. 283-285.

Fulgosi, A.. \& $\dot{Z}_{\text {AJA }}$, B. Information transmission of 3.1 bits in absolute identification of auditory pitch. Bulletin of the Psychonomic Society. 1975, in press.

GARNER, W. R. An informational analysis of absolute judgments of loudness. Journal of the Experimental Psychology, 1953, 46, 373-380.

Pollack, 1. The information of elementary auditory displays. II. Journal of the Acoustical Society of America. 1953, 25. 765-769.

LiCKLIDER, J. C. R. Basic correlates of the auditory stimulus. In S. S. Stevens (Ed.), Handbook of experimental psychology. New York: Wiley. 1951.

(Received for publication August 1, 1975.) 